\title{
Proposition d'un modèle couplé pour la simulation conjointe des écoulements de surface et des écoulements souterrains sur un bassin hydrologique
}

\section{Suggestion for a coupled model of surface and groundwater simulation on a watershed}

\author{
E. Ledoux \\ École des Mines de Paris \\ Centre d'informatique \\ géologique
}

G. Girard

ORSTOM

Service hydrologique

\author{
J.P. Villeneuve \\ Université du Québec \\ INRS Eau
}

La société hydrotechnique de France a décerné en 1983 le Prix Henri Milon à Monsieur Emmanuel Ledoux, pour son mémoire intitulé : Modélisation intégrée des écoulements de surface et des écoulements souterrains sur un bassin hydrologique.

L'article ci-après reflète l'œuvre, couronnée par le jury, qui représente une voie originale et très intéressante prenant en compte, dans une modélisation à l'échelle d'un bassin hydrologique, à la fois les phénomènes superficiels et les phénomènes souterrains avec leurs interactions.

La grande majorité des questions qui sont posées en matière de ressource en eau concerne des systèmes hydrologiques où l'eau de surface et l'eau souterraine sont étroitement associées. En effet, pour un grand nombre de régions du globe, la conjugaison de facteurs climatiques et géologiques favorables permettent l'alimentation régulière de réservoirs souterrains à partir des précipitations, ce qui assure une disponibilité continue en eau à la fois dans les nappes et dans les rivières. Ce schéma s'applique par exemple à la France mais également à tout bassin sédimentaire en climat tempéré.

Il existe cependant des exceptions. Ainsi, en zone aride, l'irrégularité des précipitations interdit la plupart du temps une alimentation des nappes par infiltration sur des surfaces étendues et la limite à des recharges temporaires le long du lit des oueds à l'occasion des crues. La ressource en eau est dans ce cas essentiellement souterraine. A l'opposé, dans les régions telles que le Bouclier Canadien, l'absence de réservoirs aquifères profonds, la régularité des précipitations ainsi que l'enneigement contribuent à maintenir l'eau à la surface du sol; le transport étant assuré par un réseau hydrographique dense doté de très nombreux lacs. L'étude de ces cas extrêmes peut justifier l'emploi d'outils de simulation purement souterrains ou inversement purement superficiels.
Cependant, en règle générale, il apparaît qu'hydrologues et hydrogéologues doivent travailler ensemble et qu'un remède doit être apporté à ce que certains auteurs ont appelé « hydroschizophrénie ".

Cette séparation n'existe en fait qu'au niveau des méthodes car il est bien certain qu'un modélisateur des écoulements souterrains doit estimer l'alimentation des nappes qui apparait alors comme une entrée de son modèle. De même, les modèles de bascin versant de surface pennent en compte un terme d'alimentation des nappes qui sera transformé en débit de base, mais la compatibilité des valeurs introduites pour ce terme, après calibrage du modèle, avec le comportement hydrodynamique des nappes n'est pas assurée.

Le travail qui est présenté ici, fruit d'une collaboration étroite entre le Service hydrologique de l'ORSTOM, l'Institut national de la recherche scientifique-Eau au Québec et l'Ecole des Mines de Paris, a pour ambition de tenter de combler cette lacune qui semble ainsi exister au niveau des méthodes en proposant au modélisateur un outil capable de prendre en compte simultanément et avec un degré de conceptualisation comparable, les maillons du cycle de l'eau entre les précipitations et les débits aux exutoires. 

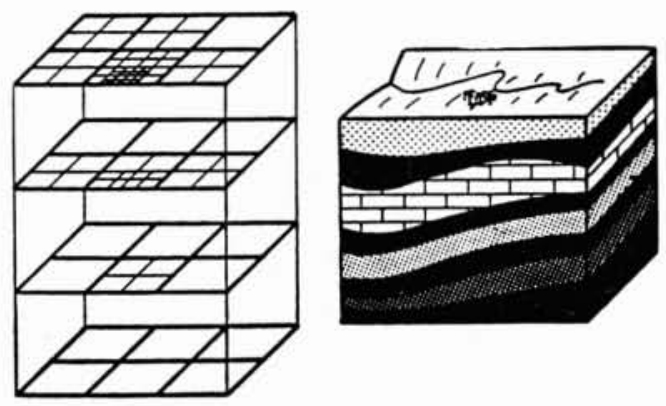

Figure 1 - Principe de la schématisation multicouche
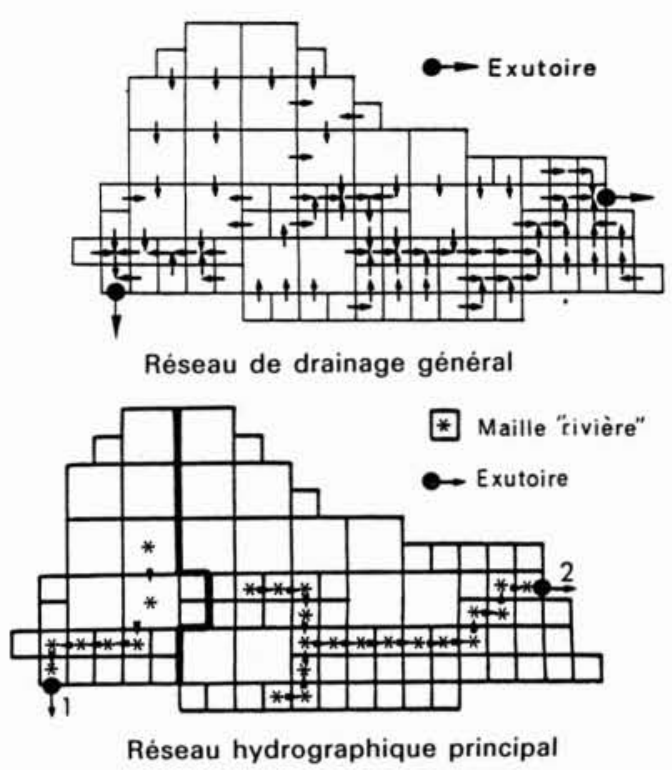

Figure 2 - Structure du réseau de drainage superficiel
L'aboutissement de ce travail se matérialise par la réalisation de programmes informatiques constituant le "modèle couplé " dont l'objectif est la simulation conjointe des écoulements de surface et des écoulements souterrains sur un bassin hydrologique (Ledoux, Girard, Villeneuve, 1980).

Le présent article a pour objectif de présenter selon deux parties distinctes d'une part un exposé des principes de fonctionnement du modèle, d'autre part un exemple d'application.

\section{Principe et conception du modèle couplé}

Le modèle couplé a été conçu avec le souci de parvenir à une représentation aussi phénoménologique que possible d'un système hydrologique. Ce vœu n'a pu cependant être réalisé qu’à des degrés divers. Certains mécanismes élémentaires du cycle de l'eau dont la connaissance est éprouvée de longue date, tels que ceux régissant les écoulements souterrains en milieu poreux, apparaitront ainsi comme très détaillés. D'autres plus complexes et moins bien connus seront traités globalement par des modèles de type paramétrique; ce sera par exemple le cas des mécanismes d'infiltration dans le sol.

La caractéristique essentielle de la méthode proposée est de chercher à décomposer la chaîne des mécanismes constituant le cycle de l'eau en étapes qui seront considérées, aussi longtemps que cela sera possible, comme indépendantes, et dont les manifestations seront contrôlables séparément. De la sorte seront réunis à la fois les avantages évidents d'une simulation globale d'un système hydrologique et la faculté de vérification de la validité de certains mécanismes internes au système.
Le modèle est articulé en quatre parties principales concernant :

- l'élaboration de la structure;

- le bilan hydrique des précipitations ou production;

- le transfert dans la zone non saturée;

- enfin les transferts conjoints superficiels et souterrains.

\subsection{Définition de la structure du système hydrologique}

La représentation des écoulements au moyen du modèle couplé nécessite tout d'abord la définition de la structure du système hydrologique, issue des observations de terrain.

\subsubsection{Le domaine d'étude}

A l'échelle régionale le domaine d'étude est la réunion d'un domaine superficiel englobant un ou plusieurs bassins versants complets ou non, et d'un domaine souterrain rassemblant des formations géologiques aquifères ayant éventuellement des relations entre elles ainsi qu'avec le domaine de surface. Un tel système est évidemment ouvert et les échanges avec le milieu extérieur sont de différentes catégories :

- échanges avec l'atmosphère;

- sorties par les exutoires superficiels;

- apports latéraux en limite du domaine de surface;

- apports latéraux en limite du domaine souterrain.

\subsubsection{Discrétisation spatiale du domaine}

La définition de la structure est fondée sur la discrétisation spatiale du domaine en éléments auxquels sont rattachées les caractéristiques physiques aussi bien superficielles que souterraines du milieu. Le mode de discrétisation adopté est une généralisation de la schématisation multicouche utilisant des mailles carrées emboîtées, devenue classique en hydrogéologie (Armisen et al. 1975. Ledoux, 1975, 1978). 


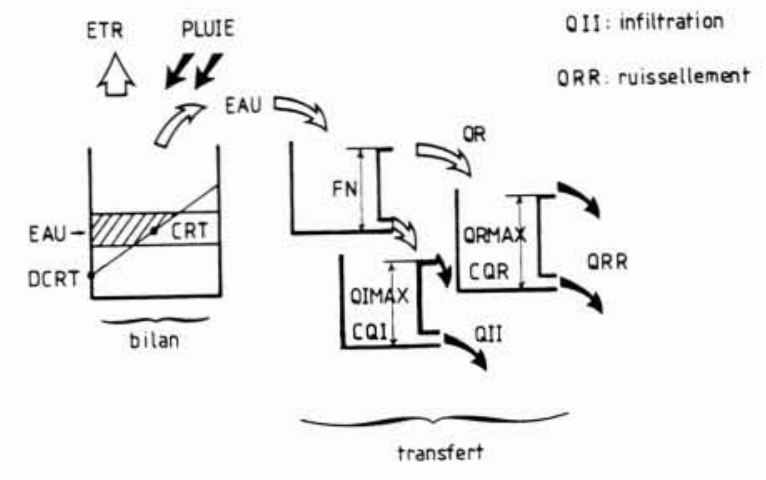

Figure 3 - Principe de fonctionnement d'une fonctionproduction

Le modèle couplé distingue, d'une part une couche supérieure dite de surface où s'effectue le partage de l'eau disponible en infiltration et ruissellement, ainsi que le transfert superficiel; d'autre part un nombre variable de couches profondes, sièges d'écoulements souterrains, éventuellement connectées hydrauliquement entre elles, figurant la succession verticale des niveaux aquifères telle qu'on peut la rencontrer dans les bassins sédimentaires constituant le cadre habituel des études de ressources en eau ( fig. 1).

\subsubsection{Définition du réseau de drainage superficiel}

Selon la discrétisation de l'espace évoquée précédemment, le réseau de drainage de surface revêt la forme d'une ou plusieurs arborescences qui sont définies sans ambiguité par la connaissance pour chaque maille de la surface d'une direction unique de vidange. Ce réseau de drainage est ensuite classé en deux catégories selon qu'il s'agit d'une maille appartenant au réseau hydrographique principal ou bien à la zone de ruissellement pur ( fig. 2).

- réseau hydrographique principal: les mailles appartenant au réseau hydrographique principal sur lesquelles ont lieu les échanges à double sens avec le domaine souterrain sont appelées "mailles rivières ". A chaque exutoire de ce réseau se trouve correspondre un bassin versant du domaine superficiel représenté par un arbre d'écoulement unique, sur lesquels seront effectués ultérieurement les transferts de l'eau en rivière.

- réseau d'écoulement de la zone de ruissellement pur: les mailles du domaine superficiel non sélectionnées comme mailles-rivières se classent en deux catégories :

- celles qui appartiennent à une portion du réseau de drainage général dont l'exutoire ne fait pas partie du réseau hydrographique principal. Elles forment alors un ou plusieurs bassins dont les écoulements ne seront pas complètement simulés, le modèle se limitant au calcul de l'infiltration en vue de l'alimentation des nappes sousjacentes.
- celles qui font parties du réseau de drainage général dont l'exutoire est une maille-rivière. Ces mailles constituent dans ce cas un "sous-bassin de calcul" dont l'exutoire est une maille-rivière sur lequel seront effectués les calculs de l'infiltration et du transfert jusqu'à la rivière indépendamment des écoulements souterrains.

Cette subdivision en deux types de mode de drainage est motivée par un souci d'allègement des calculs, en n'utilisant les possibilités complètes du modèle qu'aux endroits où cela est réellement nécessaire. Différentes configurations sont possibles, notamment les configurations extrêmes. Un problème où l'on ne s'intéresserait qu'à des écoulements souterrains déconnectés du réseau hydrographiques ne comporterait ainsi pas de mailles rivières, seul le calcul de l'infiltration étant requis. A l'opposé, un échange nappe-rivière sur l'étendue du domaine de surface est possible en affectant l'ensemble du réseau de drainage à des mailles-rivières.

\subsubsection{Définition des caractéristiques physiographiques}

Les données physiographiques introduites sur chaque maille de la surface concernent :

- l'altitude minimale du réseau de drainage, utilisée ultérieurement pour le calcul des temps de transfert;

- la nature des sols présents sur chaque maille, exprimée en fraction de superficie occupée par chaque catégorie de sol; la distinction en catégories reposant sur la manière dont doit être effectué le bilan hydrique.

\subsection{Calcul du bilan hydrique}

Le calcul du bilan hydrique, effectué au moyen des fonctions production, a pour objectif la répartition de la lame d'eau disponible au sol entre le ruissellement, l'évaporation, l'infiltration et la quantité stockée dans le sol.

Ce calcul est mené suivant le pas de temps des données des précipitations, pour chacun des postes pluviométriques définissant une zone météorologique et pour chacune des catégories de sol introduites avec la structure.

\subsubsection{Les zones météorologiques}

Selon les disponibilités en données de précipitation, chaque maille de la surface est affectée à un poste pluviométrique définissant ainsi un certain nombre de zones météorologiques caractérisées par une lame d'eau disponible au sol à chaque pas de temps. Selon l'étendue du domaine, il peut être également nécessaire de moduler la définition de ces zones en fonction de la variabilité de l'évapotranspiration potentielle.

\subsubsection{Les fonctions production}

Chaque fonction production, correspondant à une catégorie de sol, fonctionne selon un mécanisme adapté par l'utilisateur à la nature du problème. Il est fait appel à des modèles paramétriques simples appartenant à la famille des modèles à réservoirs, car les mécanismes physiques régissant le bilan hydrique sont tellement complexes que leur prise en compte à l'échelle du domaine d'étude par un modèle phénoménologique conduirait à une trop grande lourdeur d'application. Bien que para- 
métrique, il convient de noter que l'approche globale par fonction production est une approche robuste en ce sens que, tout au moins lorsqu'il s'agit de conditions d'application analogues, la transposition pure et simple des valeurs des paramètres d'une application à une autre conduit à une première évaluation satisfaisante du bilan hydrique.

La figure 3 illustre le principe de fonctionnement d'une fonction production de type standard proposée par le modèle couplé.

Le bilan hydrique est effectué dans le réservoir de bilan à chaque pas de temps en fonction de la lame d'eau précipitée $P$, de l'évapo-transpiration potentielle ETP et de l'état du stock en eau $R$ du réservoir. La quantité EAU disponible pour l'écoulement est alors déterminée par deux paramètres DCRT et CRT représentant les niveaux minimal et moyen du réservoir sol. Le premier réservoir de transfert a ensuite pour tâche de répartir l'eau disponible en une lame ruisselée $Q R$ est une lame infiltrée $Q I$ en fonction d'un seuil d'infiltration $F N$. Deux autres réservoirs de transfert apportent éventuellement un retard à ces quantités, selon un mécanisme de vidange exponentielle avant de les introduire dans le système hydrologique.

\subsection{Le transfert dans la zone non saturée}

La quantité d'eau mise en circulation dans le système ayant été déterminée au moyen des fonctions production traduisant le comportement du sol superficiel, la zone non saturée profonde est susceptible de moduler l'infiltration, selon la profondeur de la première nappe souterraine et selon les caractéristiques hydrodynamiques du milieu qui la constitue.

Etant donné qu'il est difficile sinon impossible, de recueillir des informations à l'échelle régionale sur la répartition verticale de la perméabilité de la zone non saturée, il est proposé d'opérer une modélisation de nature paramétrique mise en œuvre sur des zones homogènes définies à partir des mailles de la surface.

Des études portant sur la relation à l'échelle régionale (Besbes, 1978) entre l'infiltration dans le sol et l'alimentation des nappes ont montré que la fonction de transfert dans la zone non saturée pouvait être assimilée au modèle à réservoirs en cascade de Nash (fig. 4).

Un tel modèle dépend de deux paramètres : le nombre $N$ de réservois reflétant l'épaisseur de la zone non saturée, supposée décomposée en $N$ couches horizontales homogènes, la constante de temps de vidange des réservoirs caractérisant la vitesse de percolation verticale de l'eau. Bien entendu ces paramètres doivent être précisés par calibrage du modèle, notamment au moyen des historiques piézométriques.

\subsection{Les transferts conjoints superficiels et souterrains}

\subsubsection{Le transfert souterrain}

Ce transfert est basé sur l'intégration numérique de l'équation de diffusivité régissant les écoulements en milieu poreux. La schématisation multicouche adoptée distingue des niveaux dits aquifères, subhorizontaux, sièges d'écoulements en nappe, séparés par des niveaux dits semi-perméables où les circulations sont faibles mais toutefois capables d'assurer des transferts notables entre les aquifères à l'échelle régionale. Le modèle de transfert souterrain considère que les écoulements sont bidimensionnels dans les aquifères et monodimensionnels subverticaux dans les semi-perméables.

La traduction pour chaque maille du principe de conservation de la masse d'eau au cours du mouvement régi par la loi de Darcy en fonction de la charge moyenne
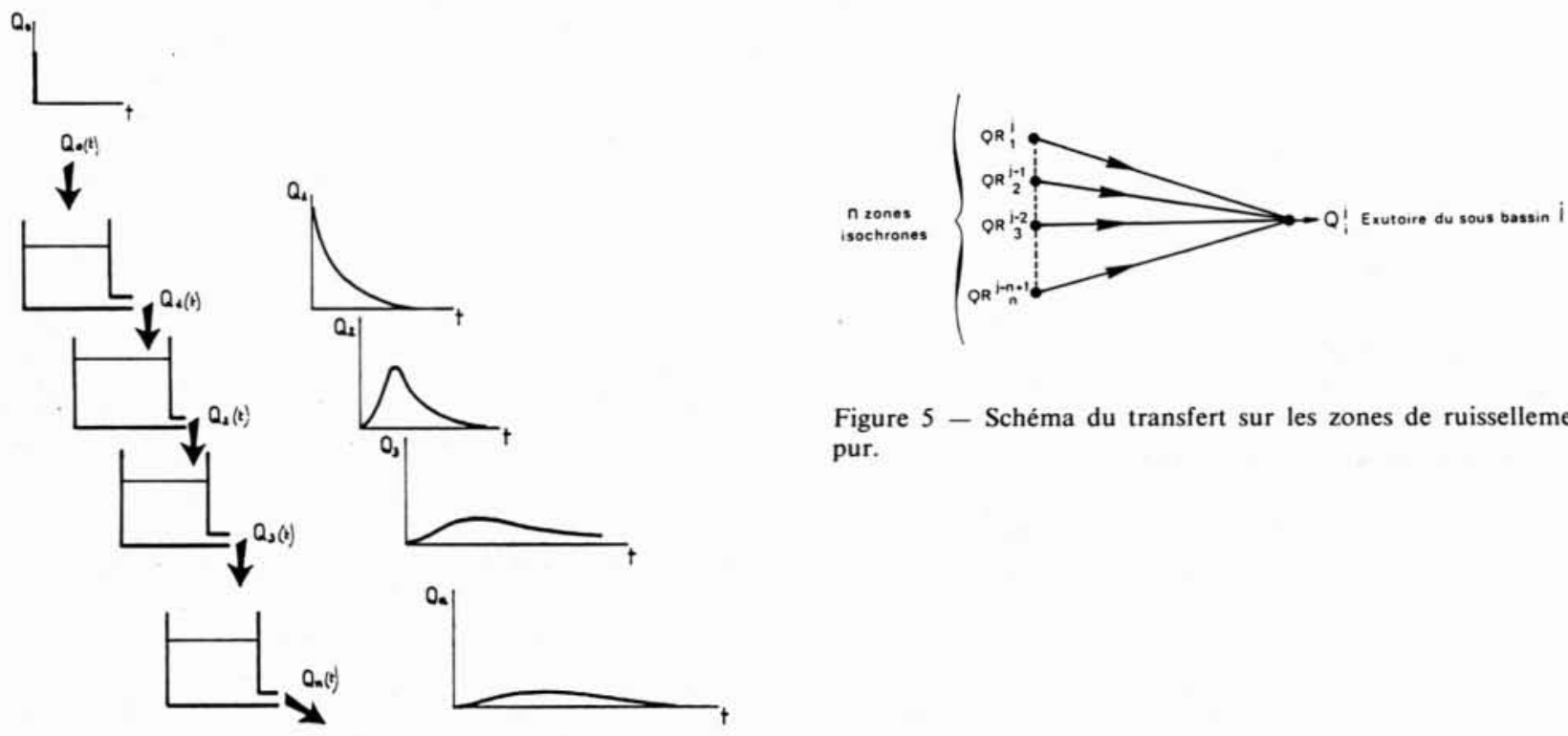

Figure 5 - Schéma du transfert sur les zones de ruissellement pur.

Figure 4 - Principe de modélisation du transfert dans la zone non saturée. 
sur chaque maille et des valeurs discrétisées des paramètres hydrodynamiques conduit à un système d'équations différentielles du premier ordre par rapport au temps que l'on résout par une méthode itérative.

\subsubsection{Le transfert sur les zones de ruissellement pur}

L'eau disponible pour le ruissellement à l'issue des fonctions production est acheminée à l'exutoire de chaque sous-bassin de calcul, c'est-à-dire jusqu'au réseau hydrographique principal selon un algorithme basé sur la notion d'isochronisme (fig. 5).

Le volume ruisselé $Q_{i}$ au pas de temps $j$ à l'exutoire du sous-bassin de calcul $i$ est obtenu par la formule :

$$
Q_{i}^{\prime}=\sum_{k=1}^{n} Q R_{k}^{j-k+1}
$$

où $Q R_{k}^{j-k+1}$ représente le volume disponible pour le ruissellement au pas de temps $j-k+1$ sur la zone isochrone de numéro $k, n$ étant le nombre total de ces zones relativement au sous-bassin de calcul considéré.

Remarquons qu'à ce niveau le transfert de l'eau est effectué globalement jusqu'à la maille rivière exutoire du sous-bassin de calcul, ce qui implique que le débit ne peut être calculé qu'à cet exutoire, à l'exclusion de tout autre point intermédiaire. De plus, le calcul considère que les écoulements en provenance des zones isochrones s'effectuent en parallèle, c'est-à-dire sans possibilité de laminage d'une crue au passage d'une zone à l'autre. Enfin l'eau ruisselant en un point du sous-bassin ne peut s'infiltrer en aval le long de son trajet vers l'exutoire. Ce cas a déjà été pris en compte au niveau des fonctions production et sera repris lors de l'écoulement en rivière.

\subsubsection{Le transfert dans le réseau hydrographique principal}

Le transfert en rivière achemine l'eau produite par ruissellement à l'exutoire des sous-bassins de calcul vers les exutoires des différents bassins en tenant compte des échanges nappes-rivières. L'algorithme de transfert considère le réseau hydrographique principal comme une arborescence de réservoirs ou biefs se vidant les uns dans les autres de l'amont vers l'aval suivant une loi de tarissement exponentielle.

Les biefs sont établis par regroupement des maillesrivières offrant à l'eau la même durée de trajet jusqu'à l'exutoire, une fois effectué le choix d'un pas de temps pour le calcul, qui peut être différent du pas de temps des précipitations (fig. 6). Le transfert porte sur le stock en eau total d'un bief et non sur le stock individuel de chaque maille-rivière, il en résulte, qu'une fois le transfert réalisé, le volume dans chaque bief doit être à nouveau réparti dans les mailles le constituant en vue de l'évaluation des échanges nappes-rivières.

A l'opposé du cas de ruissellement pur, le débit peut être simulé en n'importe quel point du réseau hydrographique principal, permettant par exemple la comparaison avec les données de différentes stations hydrométriques.

\subsubsection{La relation nappes-rivières}

Cette relation règle au niveau du réseau hydrographique principal la liaison entre le transfert souterrain et le transfert superficiel. Le couplage est effectué à chaque pas de temps entre une maille-rivière et les mailles des couches souterraines qui lui correspondent verticalement. Selon l'état hydraulique du système, deux situations sont possibles (fig. 7).

- Situation $1:$ la rivière et la nappe sont connectées hydrauliquement, la rivière imposant son niveau à la nappe moyennant une certaine perte de charge à travers son lit. Le sens de circulation de l'eau peut éventuellement varier avec le temps.

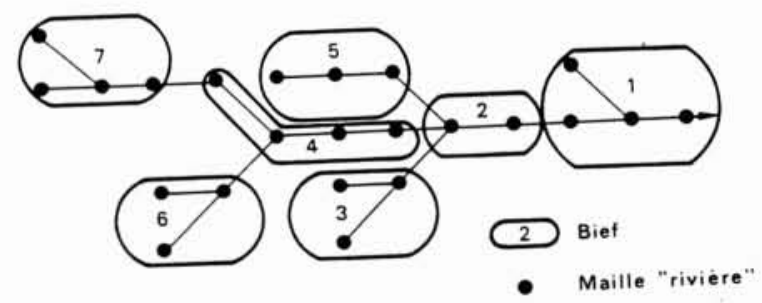

Figure 6 - Principe de constitution des "biefs " sur le réseau hydrographique principal.

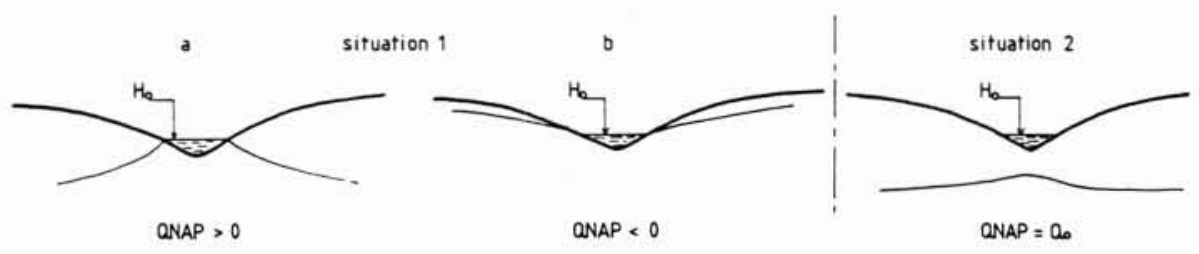

Figure 7 - Principe de la relation nappe-rivière. 

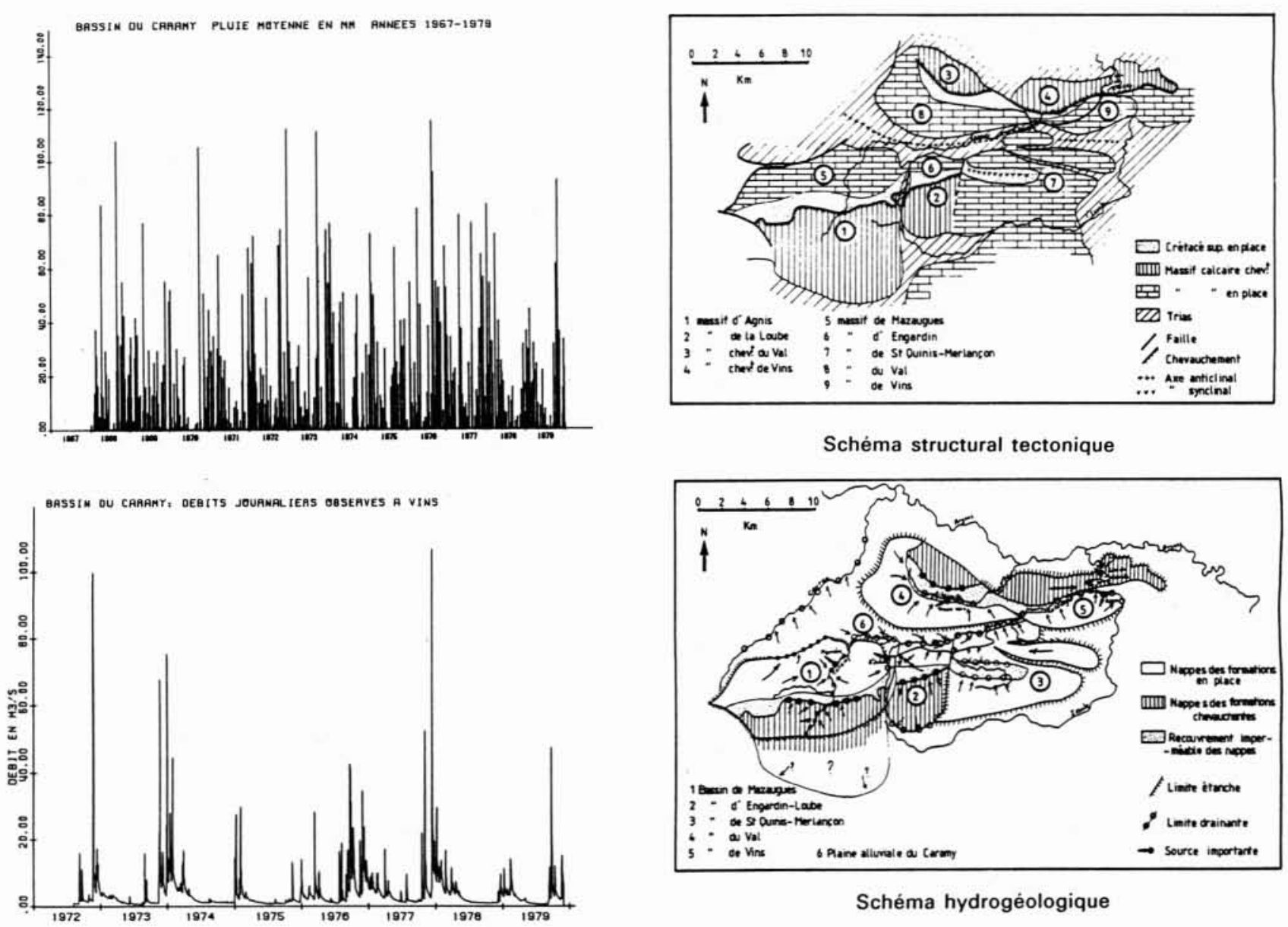

Schéma hydrogéologique

Figure 8 - Débits moyens journaliers et pluviométrie observés sur le bassin du Caramy.

Figure 9 - Structure géologique et hydrogéologique du bassin du Caramy.

- Situation 2 : la rivière et la nappe sont déconnectées par la pésence d'une zone non saturée. Le modèle considère dans ce cas que le transfert s'effectue dans le sens rivière-nappe avec une valeur donnée du débit.

Remarquons que les variations annuelles de régime hydraulique peuvent provoquer des changements de situation de la relation nappe-rivière; il est de plus nécessaire de tenir compte pour le calcul de l'infiltration de la quantité d'eau disponible en rivière. La combinaison de ces différents mécanismes aboutit à une formulation non linéaire de cette relation.

\subsection{Architecture du modèle}

Les programmes ont été conçus de façon modulaire de façon à permettre un traitement découplé des différents transferts aussi longtemps que cela est possible, ainsi qu'une adaptation aisée du modèle au problème traité.

Un premier programme (GEOCOU) concerne la définition de la structure du système qui assurera la description des caractéristiques physiques des aquifères et des caractéristiques physiographiques des bassins versants.

Un second programme (MODSUR) se charge d'établir les bilans hydriques à partir des caractéristiques de la structure et conduit à une première évaluation de l'écou- lement permettant un précalibrage du bilan hydrologique global.

Enfin le dernier programme (MODCOU) réalise la simulation conjointe des transferts superficiels et souterrains, et calcule les débits ainsi que les variations de l'état piézométrique des nappes.

\section{Exemple d'application du modèle couplé : Le bassin du Caramy}

Plusieurs applications ont été tentées sur des bassins français ou étrangers présentant une gamme de superficie de quelques dizaines d'hectares à plusieurs milliers de kilomètres carrés, ainsi qu'une large variété de structures géologiques et hydrologiques.

L'exemple du bassin du Caramy situé dans le département du Var dans le midi de la France constitue par ses caractéristiques physiographiques très hétérogènes une bonne démonstration des propriétés du modèle couplé.

Cette étude entreprise pour le compte de la Direction départementale de l'agriculture du Var a bénéficié de la participation de plusieurs organismes notamment le Ser- 
vice régional d'aménagement des eaux d'Aix-en-Provence et la Compagnie Aluminium-Péchiney.

Le travail concerne une région d'environ $250 \mathrm{~km}^{2}$ qui draine des reliefs relativement importants $(1000 \mathrm{~m})$ et bien arrosés, jusqu'au réservoir de Carces servant à l'alimentation en eau de Toulon. Une abondante pluviométrie (moyenne supérieure à $1000 \mathrm{~mm}$ sur 1968 à 1979) ainsi qu'une distribution irrégulière au cours de l'année produisent un débit de base soutenu $\left(0,5\right.$ à $\left.1 \mathrm{~m}^{3} / \mathrm{s}\right)$ et des crues parfois spectaculaires $\left(100 \mathrm{~m}^{3} / \mathrm{s}\right)$ (cf. fig. 8 ).

L'étude lithostratigraphique conduit à distinguer deux formations aquifères principales dans le Lias inférieur et dans le Jurassique moyen et supérieur constituées de calcaires et dolomies à la fois fissurés et poreux, souvent karstifiés.

Une activité tectonique conjugant des plissements et des chevauchements d'extension E-W et des failles de direction N-S a abouti à l'organisation du domaine en six bassins hydrogéologiques relativement indépendants drainée par la rivière Caramy et quelques ruisseaux affluents (cf. fig. 9).

\subsection{Mise en auvre du modèle couplé}

\subsubsection{Définition de la structure}

Le domaine superficiel a été décrit par 615 mailles carrées de $1250 \mathrm{~m}, 625 \mathrm{~m}$ et $312,5 \mathrm{~m}$ de côté dont 207 maillesrivières figurant le tracé de Caramy et de ses affluents (fig. 10).

Le bilan hydrique a été réalisé au pas de temps journalier à partir des données suivantes :

- précipitations journalières en quatre postes pluviométriques;

- évapotranspiration potentielle décadaire uniforme sur le domaine (Penman);

- cartographie des formations géologiques affleurantes.
L'emploi de 5 fonctions production a permis la distinction des :

- sols de plaine alluviale,

- sols calcaires perméables,

- sols calcaréo-dolomitiques perméables,

- sols imperméables argilo-gréseux,

- sols imperméables marno-calcaires.

Le domaine souterrain constitué de six bassins hydrogéologiques a été représenté par neuf unités aquifères éventuellement connectées les unes aux autres, discrétisées selon 1000 mailles carrées de $1250 \mathrm{~m}, 625 \mathrm{~m}$ et $312,5 \mathrm{~m}$ de côté (fig. 11). Les paramètres hydrodynamiques des aquifères, mesurés en de rares points, ont été déduits en première estimation d'après les résultats d'études hydrogéologiques antérieures. Leur extension au différentes mailles du domaine souterrain a fait l'objet d'un calibrage du modèle.

\subsubsection{Calibrage du modèle}

On disposait pour ce calibrage :

- de séries hydrométriques journalières en deux stations à l'amont et à l'aval du bassin sur la période 1972-1979; - de mesures piézométriques en trois points, irrégulièrement réparties dans le temps, la plupart recueillies à l'occasion d'essais de dénoyage de travaux miniers.

Le calibrage du modèle réalisé essentiellement sur les débits en rivière, a porté sur le réglage des points suivants :

- synchronisme des crues observées et calculées, déterminé par le choix du temps de concentration du bassin; - forme des crues dues au ruissellement, influencée par les deux paramètres du réservoir de transfert des fonctions production;

- débit de base et tarissement des nappes liés au paramètre d'infiltration des fonctions production et aux paramètres hydrodynamiques des nappes.

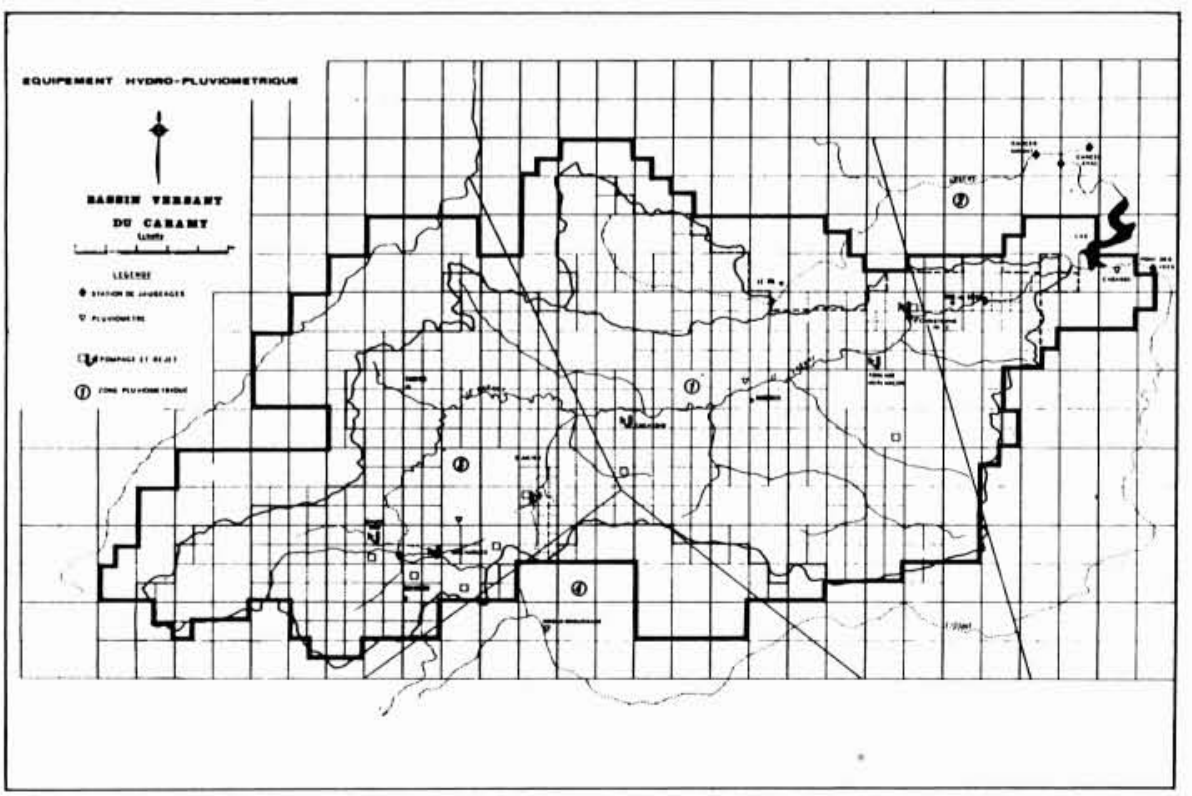

Figure 10 - Discrétisation du domaine superficiel 


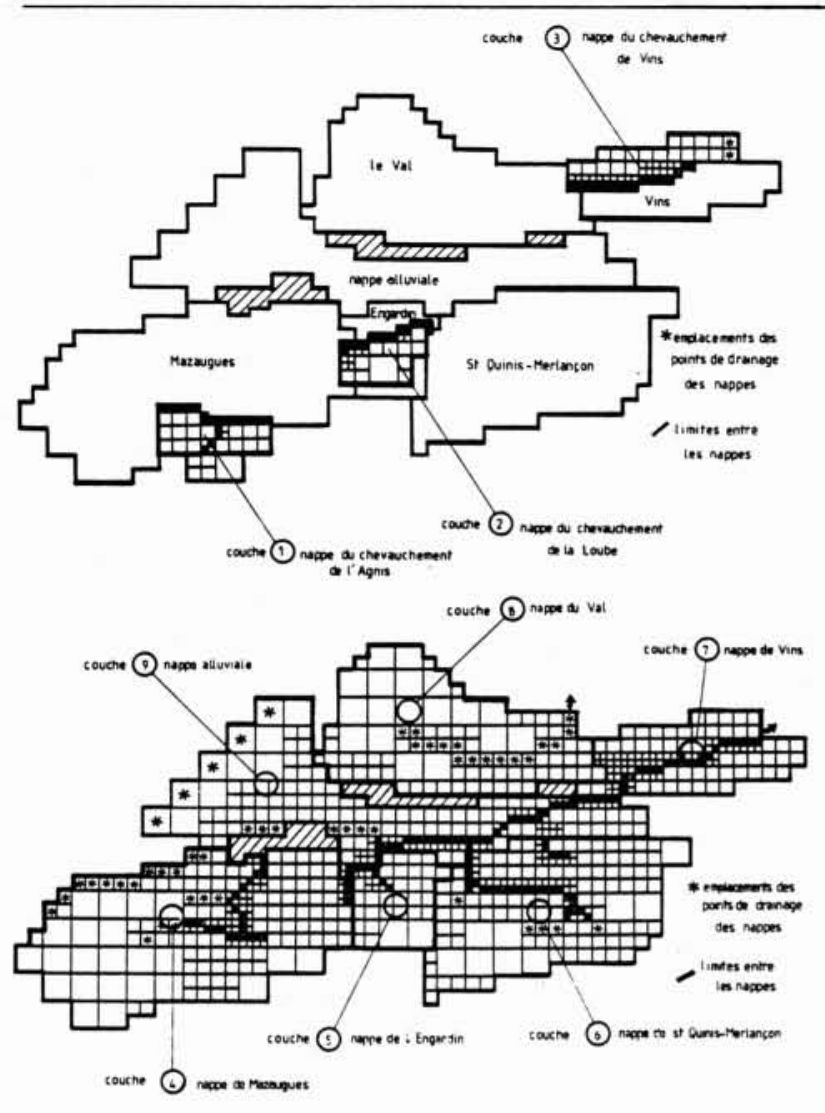

Figure 11 - Discrétisation du domaine souterrain.

\subsection{Résultats des simulations}

Deux types de simulations ont été effectuées; au pas de temps décadaire en vue d'obtenir un calibrage global pluriannuel, puis au pas de temps journalier pour l'étude d'épisodes de crues particuliers.

\subsubsection{Simulations au pas de temps décadaire}

Sur l'ensemble de la période de simulation (1968-1979), les volumes écoulés annuellement aux deux stations hydrométriques se répartissent comme suit (en millions de $\left.\mathrm{m}^{3}\right)$ :

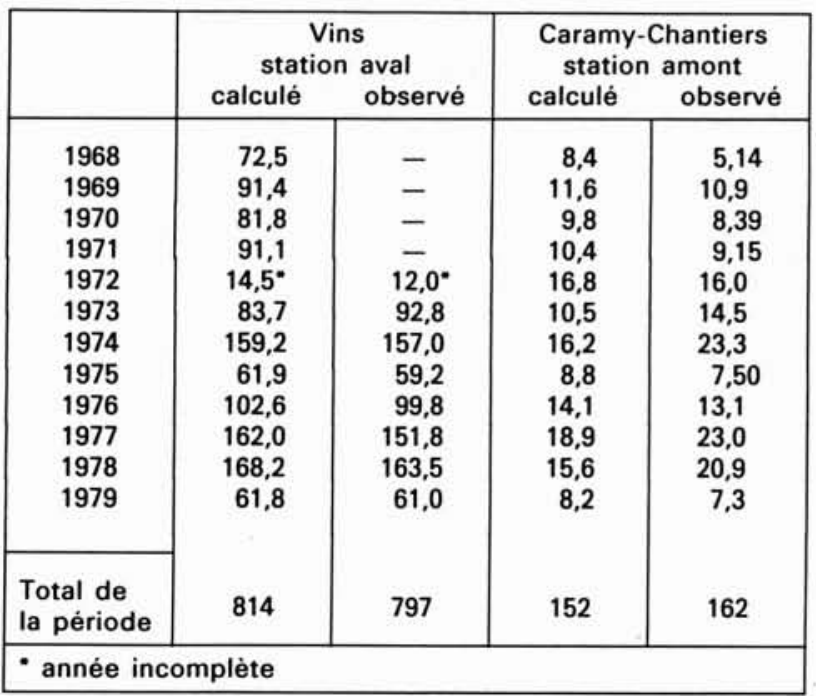

On constate une bonne reproduction par le modèle des volumes établis sur l'ensemble de la période. L'écart annuel relatif n'excède pas en général $10 \%$ sauf pendant 1972 où les observations sont d'ailleurs incomplètes.

Les figures 12 et 13 montrent les variations décadaires du débit aux deux stations, ainsi que la reconstitution d'une série piézométrique influencée par une exploitation minière (fig. 14).

\subsubsection{Simulation au pas de temps journalier}

Conservant les paramètres réglés sur les simulations décadaires, on a effectué un calcul au pas de temps journalier sur la période janvier-février-mars 1978 (fig. 15). Les résultats du modèle sont en bon accord avec les observations, notamment en ce qui concerne la reproduction d'une crue exceptionnelle dépassant $100 \mathrm{~m}^{3} / \mathrm{s}$.

Le modèle ainsi calibré a été utilisé pour la simulation et la prévision des volumes d'eau stockés dans le réservoir de Carcès, exutoire du bassin.

\section{Conclusion sur l'emploi du modèle couplé}

Les tentatives d'application du modèle couplé effectuées à ce jour permettent de dégager quelques commentaires concernant ses propriétés.

- L'adaptation à des bassins d'étendues et de caractéristiques diverses est aisée, grâce aux possibilités offertes par la discrétisation spatiale qui autorise la représentation d'hétérogénéités géologiques, physiographiques et climatiques. Le modèle s'est ainsi montré apte à simuler les écoulements sur un bassin québécois de 70 hectares occupé à $20 \%$ par un lac (bassin du lac Laflamme), sur un bassin crayeux assez homogène de $100 \mathrm{~km}^{2}$ dans le nord de la France (bassin de la Lys) ainsi que sur un bassin de $200 \mathrm{~km}^{2}$ très hétérogène dans le sud (bassin du Caramy). Une tentative à caractère anecdotique a également été faite pour simuler les débits sur la France entière. - La discrétisation spatiale facilite la transposabilité du modèle. Les termes du bilan hydrologique effectué par le modèle sont en effet fonction des paramètres physiques représentables sur la structure; or ces paramètres gardent des valeurs voisines d'un bassin à un autre si le milieu concerné est de nature comparable. Il en résulte que dans la mesure où la structure est correctement établie, le modèle doit s'adapter d'un problème à un autre avec un minimum de calibrage des paramètres. Ce problème de transposabilité des modèles à discrétisation spatiale et du " modèle couplé " en particulier fait actuellement l'objet d'une étude sur la partie française des bassins de la Meuse et la Moselle, l'objectif étant de montrer dans quelle mesure les paramètres du modèle ajustés sur la Moselle sont transposables au cas de la Meuse.

- Prenant en compte simultanément l'eau de surface et l'eau souterraine, le modèle doit être calibré à la fois sur des données de débit de sources et de cours d'eau, mais aussi sur des données piézométriques. Le modèle peut donc d'une part être plus contraint, ce qui augmente la confiance que l'on doit pouvoir lui accorder, mais il peut 


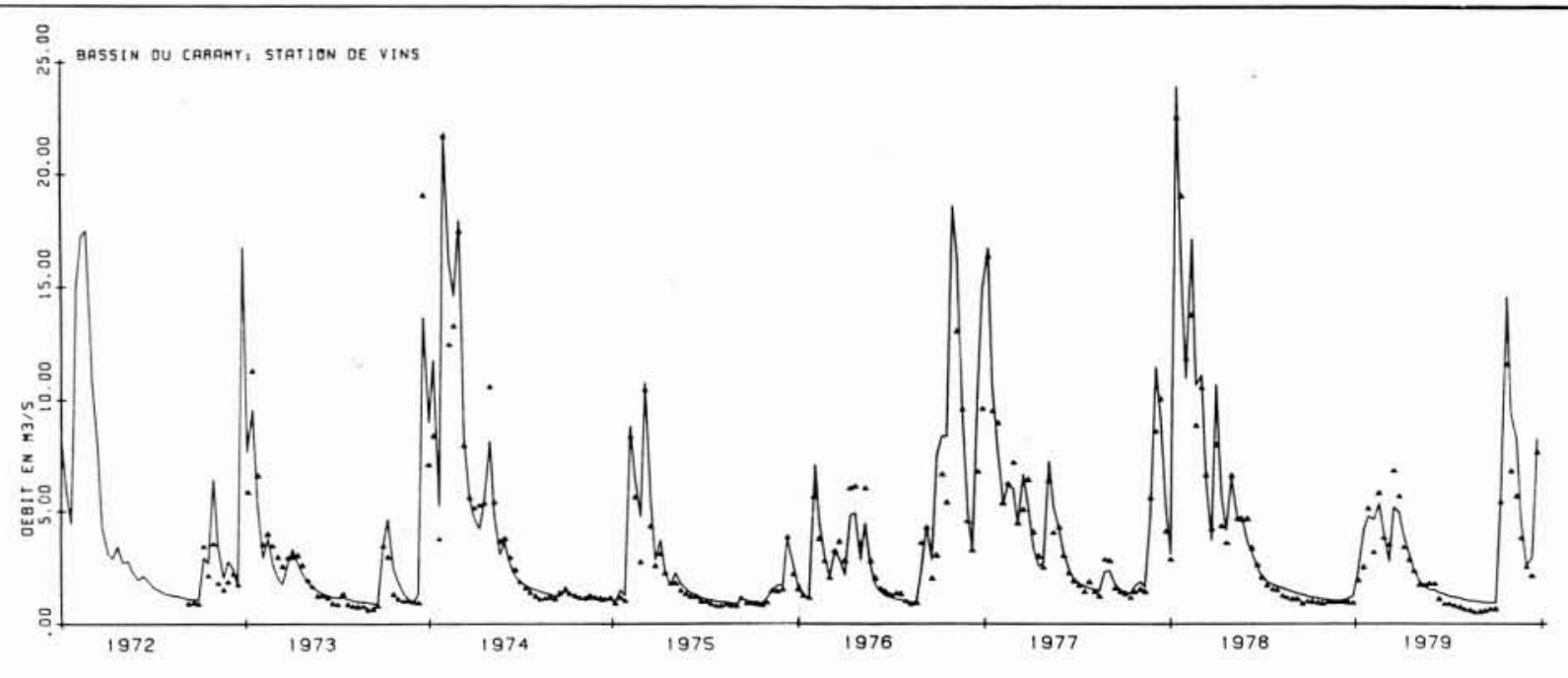

Figure 12 - Comparaison entre débit calculé $(-)$ et débit observé $(\Delta)$ à la station aval.

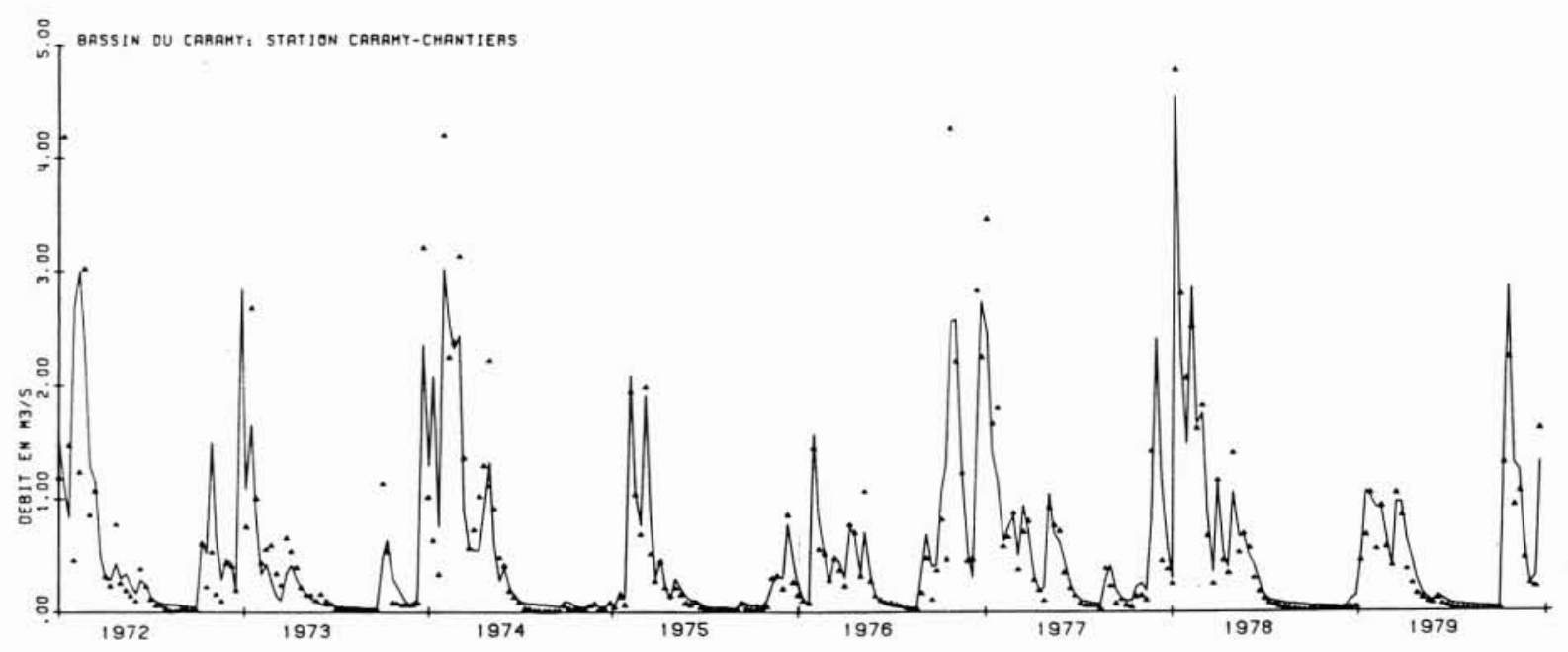

Figure 13 - Comparaison entre débit calculé $(-)$ et débit observé $(\Delta)$ à la station amont.

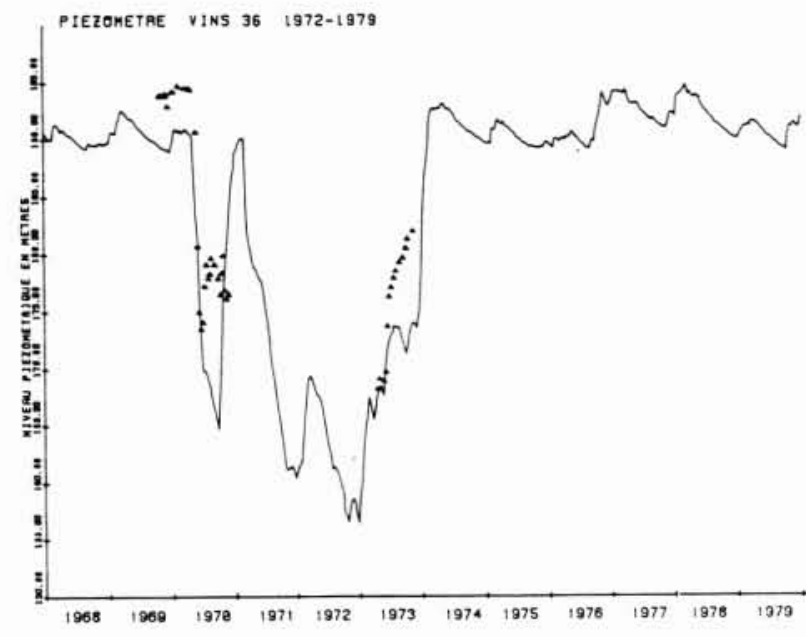

Figure 14 - Reconstitution d'un historique piézométrique.

Figure 15 - Simulation du débit au pas de temps journalier à la station aval (janvier-février-mars 1978).

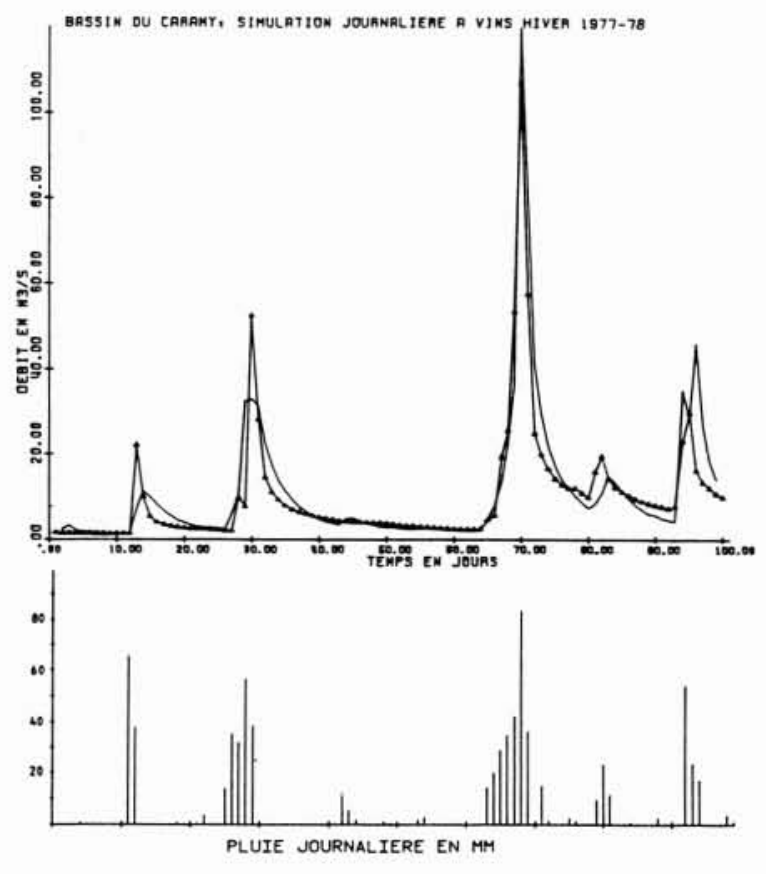


d'autre part utiliser un plus grand nombre de données, ce qui facilite le calibrage. En fin d'étude, on dispose d'un outil de simulation qui assure nécessairement un ensemble cohérent entre la pluie et les débits avec une possibilité de contrôle non seulement aux exutoires, mais encore en tout point du domaine selon la définition de la structure faite au moyen de la discrétisation spatiale.

Nous concluerons en disant que le modèle ne doit pas être considéré comme figé. L'ensemble informatique déjà réalisé constitue une structure d'accueil pouvant recevoir des perfectionnements nécessités par les besoins des problèmes pratiques. Il convient pour cela de multiplier les applications. Une évolution a d'ailleurs déjà été faite dans ce sens avec une amélioration du traitement de l'écoulement de surface permettant la prise en compte de systèmes complexes de barrages-réservoirs. Une application vient d'en être faite à l'ORSTOM sur un bassin brésilien. Une évolution future pourrait être l'extension du modèle au transport de matière accompagnant l'écoulement.

\section{Références bibliographiques}

[1] GIRARD G., MORIN G., CHARBONNEAU R. - Modèle précipitations-débits à discrétisation spatile. Cahiers ORSTOM, série Hydrologie,vol. IV. $\mathrm{n}^{\circ} 4,1972$.

[2] ARMISEN P. BESBES M., LEDOUX E., LEVASSOR A., MARSILY G. de, POITRINAL D. - Sammir : un programme mathématique de simulation des aquifères multicouches en mailles irrégulières. Congrès de Porto-Alegre (Brésil) réf. LHM/RD/75/23, 1975.

[3] LEDOUX E. - Programme NEWSAM. Principe et notice d'emploi. Rapport Ecole des Mines LHM/R75/11.

[4] MARSILY G. de, FRASNETTI J.C., LEDOUX E., TIBURTINI R., VILLENEUVE J.P. - Inventaire des ressources en eau et adéquation besoins-ressources: peut-on faire une première approche simplifiée à partir d'une modélisation complète du cycle de l'eau ? Colloque national "Les eaux souterraines et lapprovisionnement en eau de la France". Nice, 1977.

[5] MARSILY G. de LEDOUX E., LEVASSOR A., POITRINAL D., SALEM A. - Modelling of large multilayered aquifer systems : theory and applications. Journal of Hydrology, 36, 1978.
[6] BESBES M. - L'estimation des apports aux nappes souterraines. Un modèle régional d'infiltration efficace. Thèse d'Etat, Université P. et M. Curie, 1978.

[7] GIRARD G., LEDOUX E., VILLENEUVE J.P. - Modèle intégré pluie-eau de surface-eau souterraine. Société Hydrotechnique de France, session 114, novembre 1979.

[8] LEDOUX E. - Modélisation intégrée des écoulements de surface et des écoulements souterrains sur un bassin hydrologique. Thèse de Docteur-Ingénieur. Ecole des Mines de ParisUniversité Pierre et Marie Curie. Paris, 1980.

[9] GIRARD G., LEDOUX E., VILLENEUVE J.P. - Le modèle couplé : simulation conjointe des écoulements de surface et des écoulements souterrains sur un système hydrologique. Cahier ORSTOM, série Hydrologie. vol. XVIII n ${ }^{\circ} 4,1981$.

[10] VILLENEUVE J.P., GIRARD G., LEDOUX E. - Modélisation et simulation intégrées : eau de surface-eau souterraine. Eau du Québec. vol. 15, n 1, février 1982.

[11] DESCHENES J., VILLENEUVE J.P., LEDOUX E., GIRARD G. - Modélisation intégrée des écoulements de surface et souterrain : application du modèle couplé au bassin versant du Lac Laflamme au Québec; Bulletin du BRGM. section III (à paraître), 1983. 


\title{
Colloque SHF - INRA \\ Avignon 24-25 juin 1982
}

\section{Variabilité spatiale des processus de transfert dans les sols}

\author{
Spatial variability of transfer \\ processes in soil
}

Résumés des rapports - Summaries of papers

La Houille Blanche publie aujourd'hui les résumés des communications présentées au Colloque qui s'est tenu en 1982 à la Station des sciences du sol de l'Institut national de la recherche agronomique, en Avignon, sous la responsabilité de M.R. Guennelon.

Ce colloque était organisé par le groupe de travail "Dispersion en milieux poreux " de la Société hydrotechnique de France.

Les textes complets des communications ont été publiés en 1983 par I'INRA ( $\mathrm{n}^{\circ} 15$ de la collection Les colloques de I'INRA. - INRA Publications, route de Saint-Cyr, 78000 Versailles, 1983, 220 p., ISBN 2-85340-527-3).
Published in the present issue of "La Houille Blanche" are abstracts of papers submitted at the seminar held in 1982 at the Soil Sciences Station of the National Institute of Agronomics Research in Avignon, under the auspices of $\mathrm{Mr}$ R. Guennelon.

The seminar was organized by the working group on "Dispersion in a porous environment" of the Société Hydrotechnique de France.

Complete texts of the papers were published by INRA in 1983 (no. 15 of the Colloques de l'INRA. - INRA Publications, route de Saint-Cyr, 78000 Versailles, 1983. 220 p., ISBN 2-85340-527-3).
Dans toutes les disciplines qui ont affaire à des phénomènes spatialement distribués dans les milieux naturels : météorologie, hydrologie, reservoir engineering, hydrogéologie, géologie, agronomie,...,

on retrouve un même ensemble de concepts recensés sous les termes suivants :

- hétérogénéité, variabilité spatiale

- échantillonnage, représentativité

- effet d'échelle.

Une première grande catégorie de problèmes est constituée par :

- d'une part, l'estimation des moyennes spatiales et de l'incertitude qui affecte les résultats correspondants (lame d'eau sur un bassin versant, pertes de fertilisants sous une culture, réserves d'un réservoir,...);

- d'autre part, la cartographie des paramètres (pluviométrie, perméabilité, transmissivité, fissuration, caractéristiques physico-chimiques d'un sol,...); cette cartographie a souvent pour finalité le choix des valeurs des paramètres affectées aux mailles d'un modèle.
Les techniques mathématiques utilisées dans ce domaine sont celles de la géostatistique; employées d'abord en recherche minière, elles ont été progressivement étendues au "reservoir engineering", à l'hydrogéologie et l'hydrologie et, plus récemment, à l'agronomie.

Un deuxième aspect des problèmes pusés par l'hétérogénéité est l'effet d'échelle; on peut tenter de le résumer de la manière suivante. Si un même paramètre est utilisé pour définir un phénomène de même nature à deux échelles différentes (par exemple, la notion de dispersivité dans un déplacement miscible), les questions suivantes peuvent se poser: les valeurs de ce paramètre sont-elles dépendantes de l'échelle considérée ? Si oui, et il y a bien alors effet d'échelle, sait-on relier de manière rationnelle les valeurs relatives aux échelles respectives ?

Des questions analogues, souvent liées à celles que l'on se pose sur la valeur des paramètres, peuvent être évoquées sur les lois et les équations physiques utilisées (validité de l'équation de la dispersion, des lois des écoulements non miscibles quand l'échelle est celle du réservoir ou d'une portion de réservoir, par exemple). 
L'effet d'échelle peut être perçu de différentes manières : soit, directement, à travers des mesures ou des résultats obtenus à des échelles différentes (par exemple, mesure des propriétés dispersives d'une couche aquifère sur des échantillons et à l'échelle d'un périmètre expérimental, mesure des perméabilités sur des échantillons et essais de puits,...), soit par le décalage observé entre les valeurs à l'échelle de l'échantillon et de la parcelle et celles déterminées pour les mailles d'un modèle par l'ajustement sur un historique.

All disciplines involved in phenomena spatially distributed in natural environments, namely meteorology, hydrology, reservoir engineering, hydrogeology, geology and agronomy deal with an aggregate of concepts clustered together under the following generic terms :

- heterogeneity, spatial variability

- sampling, representativity

- scale effect

A first major category of problems may be broken down into two parts :

- the first part covers a valuation of spatial averages and of the uncertainty affecting the related results e.g. water line in river basins, loss of fertilize under cultivations, reserves of reservoirs etc.;

- the second part covers the mapping of the parameters (pluviometry, permeability, transmissivity, crevassing, physico-chemical properties of soils etc.). The ultimate purpose of such mapping is the choice of parameter values assigned to the grids is a model.

The mathematical techniques used in such cases are those of geostatistics. Initially utilized in mining research, they have gradually been extended to reservoir engineering. hydrogeology, hydrology, and more recently to agronomy.

A second aspect of the problems posed by heterogeneity is scale effect, which may be summed up as follows. If a given parameter is used to define a phenomenon of the same nature at two different scales (e.g. the concept of dispersiveness in a miscible displacement), the following question arises: are the values of the parameter dependent on the considered scale? If yes - in which case there is indeed scale effect - can one rationally relate the values to the respective scales?

Similar questions, frequently related to those raised about parameter values, may be mentioned in connection with the physical laws and equations used i.e. validity of dispersion equations, and the laws of non miscible flows where the scale is that of the reservoirs or of a portion of a reservoir.

Scale effect may be perceived in various ways : either directly through the measurements or results obtained at various scales (e.g. measurement of the dispersive properties of an aquifer on samples and at the scale of a test perimeter. permeability measurements on well samples and tests, etc.). Or it may be perceived through the observed discrepancy between the values at the scale of the samples and of the plots, and those determined for the grids of a model by adjustment to a historical background.

\section{Résumés - Summaries}

\section{VAUCLIN}

\section{Institut de Mécanique de Grenoble} B.P. 53 X-38041 Grenoble Cedex, France

\section{Méthodes d'étude de la variabilité spatiale des pro- priétés d'un sol}

Il est bien connu que les propriétés physico-chimiques des sols varient d'un point à un autre, d'une parcelle ou d'un bassin versant. Identifier et analyser cette variabilité nécessitent l'utilisation de concepts statistiques et géostatistiques. Modéliser les transferts implique qu'à l'aspect déterministe des équations doit s'ajouter une description stochastique des variables d'état, des paramètres caractéristiques et des conditions aux limites. En se fondant sur des exemples concrets, on présente les différentes approches utilisées pour décrire et analyser l'hétérogénéité d'une parcelle : approches de la statistique classique et de la géostatistique, théorie de la mise en facteurs d'échelle. On montre notamment l'intérêt de cette dernière tant au niveau de la réduction des données expérimentales que de la modélisation des transferts hydriques.

\section{Survey of methods for studying spatial variability of soil properties}

It is well-known that Soil physical and chemical properties vary within an agricultural field or a watershed. Studying the variability of these properties needs the use of statistical and geostatistical tools. Solving the deterministic flow equations implies a stochastic description of descriptive variables, key parameters and boundary conditions as well.

A review of different approaches (statistical and geostatistical analysis, scaling theory) more or less classically used to describe and to analyze the soil properties variability is presented through several practical examples. An emphasis is put on the scaling theory for data reduction and stochastic modelling purposes. 
G. VACHAUD*, Z. CHAABOUNI**, S. El AMANI** et M. VAUCLIN*

\section{* Institut de Mécanique de Grenoble} B.P. $53 X-38041$ Grenoble Cedex, France ** Centre de Recherches du Génie rural B.P. 10, Tunis-Ariana, Tunisie

\section{Méthodologie d'étude du bilan hydrique d'une culture} à l'échelle de la parcelle (1)

De nombreuses études théoriques et expérimentales ont montré que l'utilisation simultanée de l'humidimètre neutronique et de tensiomètres permet d'estimer très correctement le bilan hydrique d'une culture sur la verticale de mesures. La représentativité des résultats pour l'ensemble de la parcelle se heurte cependant à la variabilité spatiale des sols et de la culture, et un site de mesures ne suffit pas. A partir d'une expérimentation conduite sur une parcelle de 1 ha située au MornagTunisie (station hydroclimatologique ORSTOM/ferme INRAT), on propose une méthodologie d'implantation des sites de mesures du bilan hydrique et de son évolution dans le temps fondée sur les critères suivants :

a) Choix d'un nombre limité (et le plus faible possible) de sites permettant d'estimer valeur moyenne et variance de la consommation hydrique, en relation avec la variabilité du sol.

b) absence d'autocorrélation entre sites afin de garantir la pertinence des hypothèses de l'analyse statistique classique.

c) accès facile aux sites pour éviter la détérioration de la culture et assurer ainsi la représentativité de la mesure.

Cette méthodologie s'appuie sur les phases suivantes :

1) reconnaissance de la parcelle :

Des prélèvements gravimétriques à la tarière sur $0-100 \mathrm{~cm}$ par tranche de $20 \mathrm{~cm}$ ont été effectués à différents temps aux nœuds d'une grille $20 \times 20 \mathrm{~m}$ et/ou le long de transects afin de définir la variabilité spatiale des composants texturaux, des humidités par couches de sol et moyennes sur $0-100 \mathrm{~cm}$ et d'établir une cartographie pédologique de la parcelle.

2) choix de sites représentatifs :

L'analyse statistique des observations montre que les humidités et leurs variations temporelles suivent une loi normale de distribution. Elle met également en évidence la grande stabilité dans le temps des points caractéristiques de ces lois (valeur moyenne, valeur moyenne \pm écart-type).

L'analyse géostatistique, met en évidence, outre une anisotropie marquée, une distance d'autocorrélation de $20 \mathrm{~m}$ environ, pour les mesures d'humidité.

3) suivi du bilan hydrique :

L'analyse des données neutroniques et tensiométriques obtenues en 3 sites de mesures (représentatifs de la moyenne, de la moyenne augmentée ou diminuée de l'écart-type) situés à des distances supérieures à $20 \mathrm{~m}$ permet alors d'estimer les différentes composantes du bilan hydrique d'une culture pluviale de blé.
Large scale estimation of seasonal water uptake by wheat, considering the spatial structure of soil variability

Root water uptake by a cultivation of rainfed wheat is estimated at the scale of a I hectare field plot from a selected and limited number of neutron access tubes, at the experimental site of Mornag near Tunis, Tunisia. The structure of the soil variability of the site was first determined from a systematic prospection to a depth of $1.4 \mathrm{~m}$ using soil sampling with an auger. Two series of measurements were done: along two orthogonal transects (100 m long) with one sample each $5 \mathrm{~m}$; then all over the field $(100 \times 100 \mathrm{~m})$, with $a$ sample at the center of a $20 \mathrm{~m}$ square grid. The soil water storage from 0 to $1 \mathrm{~m}$ was found to be normally distributed. Spatial correlograms show that measurements were autocorrelated within distance of approximetly $25 \mathrm{~m}$.

Five measurements sites, each one with a neutron access tube to a depth of $1.7 \mathrm{~m}$, and with tensiometers at depth of $30,75,90$ and $130 \mathrm{~cm}$ were installed at sites selected from the cumulative probability distribution of soil water storage to represent roughly the mean value at the scale of the field, one standard deviation value and the two extrema (dryest and wettest). Weekly measurements were carried from Nov. 1981 to July 1982, using a neutron moisture meter. Finally the stationnarity of the distribution law was checked by repeating at different time of the crop cycle gravimetric sampling within the $20 \times 20 \mathrm{~m}$ grid.

\section{THIRRIOT et J. VALIANTZAS}

Institut de Mécanique des Fluides de Toulouse

Laboratoire associé au CNRS $n^{\circ} 005$

31071 Toulouse, France

Problèmes et procédés de simulation numérique des écoulements en milieu poreux hétérogène

Après avoir défini le domaine d'écoulement, les auteurs examinent en détail la question de la création d'un champ hétérogène de perméabilité. En particulier, ils étudient l'influence du choix du procédé d'interpolation sur l'altération de la fonction de distribution et sur la forme du corrélogramme.

Ils présentent ensuite les grandes lignes de l'organisation du calcul numérique.

Ils illustrent enfin leur propos par quelques exemples de calcul qui font apparaître les perméabilités du champ uniforme globalement équivalent.

\section{Problems and procedures in numerical simulation of flow in heterogeneous porous environments}

After defining the scope of flow, the authors analyse in detail the question of the generation of a heterogeneous permeability field. More specifically, they investigate the effects of choosing a process of interpolation on changes in the distribution function and on the shape of the correlograms.

They then show the major thrusts of the organization of numerical computation. Lastly the authors illustrate their argument with some typical computations that point up the permeabilities of a generally equivalent uniform field. 


\section{B. JAILLARD ET Y-M. CABIDOCHE}

\section{I.N.R.A., Laboratoire d'Agronomie \\ C.R.A. des Antilles et de la Guyane \\ Domaine Duclos \\ 97170 Petit-Bourg, Guadeloupe}

\section{Dynamique hydrique et porale dans un sol argileux gonflant}

Ce texte est un condensé de deux publications à paraître dans la revue "Science du Sol ", intitulé :

- "Etude de la dynamique de l'eau dans un sol argileux gonflant "

I - Dynamique hydrique

II - Dynamique porale

\section{Hydric and poral dynamics in heaving clay soils}

This is a condensed version of a publication to appear in the review "Science du Sol " entitled : "Study of water dynamics in heaving clay soil " comprising two parts : Hydric dynamics, and Poral dynamics.

\section{A. DiEulin, G. de MARSily, P. GOBLET}

Centre d'Informatique géologique

35 , rue Saint-Honoré

77305 Fontainebleau, France

Effet de la variabilité spatiale du champ des vitesses sur le transport miscible en milieu poreux saturé

Le transport miscible en milieu poreux hétérogène saturé est étudié théoriquement par l'utilisation du concept de mouvement de particule dans un champ de vitesses aléatoire. Il est montré que le tenseur de dispersion dépend du temps à la fois en direction et en amplitude. L'équation du transport obtenue sous certaines hypothèses est différente de l'équation classique de la dispersion. La simulation numérique de la propagation des particules illustre les propriétés du tenseur de dispersion étant donné certaines caractéristiques statistiques du milieu poreux. Ces résultats légitiment enfin l'utilisation de la fonction temporelle de dispersion dans l'interprétation des essais de traçage.

\section{Effect of the spatial variability of the velocity field in solute transport in saturated porous media}

Solute transport in heterogeneous, saturated porous media is studied theoretically by analysis of particule movements in a random velocity field. Dispersion tensor is shown to be time dependant both in direction and amplitude. A transport equation obtained in a special case is different from the classical dispersion equation. By numerical simulation of particule movements properties of the dispersion tensor have been calculated given certain statistical properties of the porous medium. Finally, these results legitimate the use of $a$ " temporal function of dispersion " in the interpretation of field tracer tests.

\section{Chantal GASCUEL-ODOUX, P. MEROT et P. CURMI}

I.N.R.A., Laboratoire de Science du Sol 65 , route de Saint-Brieuc 35042 Rennes Cedex, France

Approche de la variabilité spatiale de l'état et du transfert hydrique dans le sol

Sur un bassin versant élémentaire de dix hectares, dans la région de Rennes, la variabilité spatiale du fonctionnement hydrodynamique du sol est appréhendée à l'aide d'approches morphologiques - caractérisation détaillée et cartographie des horizons - et géostatistique. A partir de la théorie des variables régionalisées, une caractérisation statistique et une cartographie de l'état hydrique du bassin versant a été entreprise, à un moment donné de la période hivernale. Ces différentes approches, qui débutent actuellement, ont été confrontées.

Une étude spatiale de la composition isotopique de l'eau en oxygène 18 , utilisé comme traceur intrinsèque du transfert réel de l'eau dans le sol, poursuit ce travail et permettra de connaître l'hétérogénéité spatiale du fonctionnement hydrodynamique d'un petit bassin versant élémentaire, en relation avec la morphologie des sols.

Approach to spatial variability in soil condition and hydric transfer

In a unit river basin of ten hectares located in the Rennes region, the spatial variability of the hydrodynamic operation of the soil is determined by a two-fold approach: the morphological (detailed characterization and mapping the horizons) and the geostatistic approach. Based on the theory of regionalized variables, a statistical characterization and mapping of the hydric condition of the river basin has been undertaken at a given moment of the winter period. The two approaches which have just been initiated are now being confronted.

A spatial survey of the isotopic composition of oxygen 18 in water, used as intrinsic tracer of the actual transfer of water in soil, will continue the work. It will lead to determining the spatial heterogeneity of the hydrodynamic operation of a small unit river basin in relation to soil morphology.

\section{VIVILLE, B. AMBROISE et J.L. MERCIER}

Laboratoire de Géographie physique

ERA 569 CNRS, Institut de Géographie 43 rue Goethe - 67083 Strasbourg Cedex, France.

Etude de la variabilité spatiale des caractéristiques hydrodynamiques des sols dans le bassin versant du Ringelbach, Hautes Vosges (France).

Une procédure pour estimer la variabilité spatiale (tant verticale que latérale) des caractéristiques hydrodynamiques (rétention hydrique, conductivité hydraulique) des 
sols et formations superficielles est testée dans le petit bassin versant du Ringelbach ( 36 ha) à Soultzeren, dans les Hautes Vosges. Elle consiste à :

- effectuer un premier découpage du bassin en unités géomorpho-pédologiques " homogènes ";

- procéder à un échantillonnage de chaque unité, de façon :

- à déterminer si ces unités sont significativement différentes aussi du point de vue hydrodynamique (variabilité inter-unité) et à faire d'éventuels redécoupages ou regroupements d'unités;

- à estimer les variations résiduelles dans chaque unité du fait des micro-hétérogénéités locales (variabilité intraunité), ainsi que la représentativité spatiale de toute mesure ponctuelle de ces caractéristiques.

La détermination in situ de ces caractéristiques étant difficile sur pente, et de toute façon trop lourde pour être étendue à tout un bassin, les mesures de conductivité à saturation et de rétention hydrique $(\mathrm{pF} \leqslant 2.7$ : bac à sable et à kaolin; $\mathrm{pF} \geqslant 3$ : presse à membrane) sont faites en laboratoire sur des échantillons de sol non remanié $\left(250 \mathrm{~cm}^{3}\right)$. L'ajustement aux courbes expérimentales de rétention des modèles de Mualem (1976) et de van Genuchten (1980) permet une analyse statistique des résultats et un classement facile des échantillons. Ces résultats sont interprétés à l'aide des courbes granulométriques, teneurs en matière organique et densités apparentes mesurées pour chaque échantillon.

Une première série de 450 échantillons a été prélevée en une quinzaine de profils représentatifs et le long de certains transects. Leur traitement est en cours.

\section{Analysis of spatial variability of the hydrodynamic properties of soils in Ringelbach river basin, Hautes Vosges, France.}

A procedure for estimating the spatial (vertical and lateral) variability of hydrodynamic properties (water retention, hydraulic conductivity) in soils and superficial formations is being tested in the small Ringelbach catchment ( $36 \mathrm{ha}$ ) at Soultzeren, High Vosges Mountains (France). The method is:

- to perform a preliminary subdivision of the area into " homogeneous" geomorpho-pedologic units;

- to sample each unit, in order to :

- determine if these units are significantly different also as hydrodynamic units (inter-unit variability) - that can lead to a finer subdivision:

- estimate in each unit the residual variations due to local microheterogeneities (intra-unit variability) and also the spatial representativity of any local measurement of these properties.

The in situ determination of these parameters is difficult on slopes and much too complex to be applied all over a basin. Therefore, saturated conductivity and water retention curves are measured in the laboratory on undisturbed 250 $\mathrm{cm}^{3}$ soil cores $(p F \leqslant 2.7$ : sand-and-kaolin box apparatus; $p F \geqslant 3$ : pressure membrane apparatus).

Van Genuchten's function (1980) is fitted to the experimental curves to determine parameters more suitable for a statistical analysis of the results and to deduce an estimation of the corresponding unsaturated conductivity curves according to Mualem's model (1976). Results are analysed with respect to the grain size distribution and organic matter content measured for each sample.

A first set of 450 cores has been sampled in 15 representative profiles and along transects. They are being analysed with the above procedure.

\section{A. BOUCEK* et M. LIVET**}

* Laboratoire régional des Ponts et Chaussées

8-10, rue B. Palissy

63014 Clermont-Ferrand, France

** Institut de Mécanique de Grenoble

B.P. $53 X$-3804I Grenoble Cedex, France

Analyse structurale d'une pente argileuse par l'application des variogrammes

Sur le site expérimental de Salledes, deux paramètres ont été étudiés, afin de préciser la structure du terrain :

- La perméabilité à saturation $K_{s}$, évaluée à partir des essais d'infiltration:

- La teneur en eau $W$, déterminée au Laboratoire.

L'ensemble des valeurs des $K_{s}$ n'a pas de structure à l'échelle du travail; dans le cas présent, le pas de mesure est de $5 \mathrm{~m}$. Les valeurs de $W$, par contre, sont structurées et donnent la taille moyenne du phénomène, de 9 à $10 \mathrm{~m}$ pour une échelle de travail prise égale à $1 \mathrm{~m}$.

Les variogrammes expérimentaux et leur ajustement par variogramme théorique sont illustrés. Leur signification et leur représentativité sont discutées.

\section{Structural analysis of a clay slope by means of vario- grams}

Two parameters measured at the experimental station of Salledes have been analysed to study the spatial structure of the field:

- satured permeability coefficient, $K_{s}$, determined by infiltration experiments in the field:

- natural water content, $W$, determined in the laboratory.

There was no structure found for the permability values at the chosen distance of $5 \mathrm{~m}$ between the measurement points, whereas the water content measurements having a sampling distance of $1 \mathrm{~m}$ are structured with a range of 9 to $10 \mathrm{~m}$.

The experimental variograms are presented and fitted by several variogram models. Their significance and representativity are discussed.

\section{SCHWEICH, P. STENZEL et M. SARDIN}

\section{Laboratoire des Sciences du Génie chimique CNRS-ENSIC, I rue Grandville. 54042 Nancy Cedex, France}

\section{Propagation transitoire de cations échangeables dans un sable argilo-calcaire}

L'injection temporaire de cations alcalins dans un sol se rencontre dans de nombreuses situations: épandage d'engrais, injection de tensio-actifs sodiques en récupéra- 
tion assistée du pétrole... Outre l'écoulement de l'eau, l'échange d'ions avec les argiles, la dissolution du calcaire, la quantité de gaz carbonique dissous altèrent le mouvement du cation. Nous proposons ici de caractériser l'importance de ces divers phénomènes en partant de résultats expérimentaux obtenus dans une colonne de laboratoire garnie d'un sable argilo-calcaire et parcourue par un écoulement permanent d'eau. On y montre essentiellement le rôle primordial de traces de calcaire qui peuvent provoquer des retards considérables de l'élution de cations alcalins comme le sodium. Ces retards sont dus à la compétition entre l'échange d'ions et la dissolution de la calcite.

Transient moment of exchangeable cations in a sand containing clays and calcite

The transient injection of alcaline cations in a natural soil is encountered in various situations : scattering of manure, injection of sodic surfactants in tertiary oil recovery.

Besides the flow of water, the ion-exchange process with clay particles, the solubilisation of calcite, and the amount of dissolved carbon dioxide disturb the movement of the cations. The part played by these processes is studied by means of experiments performed in a laboratory column packed with a sand containing clays and calcite. A special emphasis is laid on the effects of a small amount of calcite which induces large retentions of sodium due to the competition between the ion exchange process and the solubilization of calcite.

R. GUENNELON*, A. ZEILIGUER** et A.M. de COCKBORNE*

* INRA, Station de Science du Sol

Centre de Recherches agronomiques

84140 Montfavet, France

** Chaire de Pédologie de l'Institut d'Amélioration des Sols, Moscou V-550, URSS

\section{Effets texturaux sur la porosité et la dispersion hydrodynamique}

La conductivité hydrodynamique d'un matériau dépend de ses caractéristiques granulométriques mais aussi du mode d'arrangement de ses constituants. Cet arrangement ne varie pas de façon continue et pour certaines compositions granulométriques peuvent apparaître des discontinuités qui peuvent influencer les propriétés hydriques du milieu.

\section{Texture effects on porosity and on hydrodynamic disper- sion}

The hydrodynamic conductivity of materials depends not only on grain size but on the arrangement of its constituents. The arrangement does not vary continuously, and in some grain size compositions discontinuities may appear that may affect the hydric properties of the environment.

\section{R. HAVERKAMP* et J.Y. PARLANGE**}

* Institut de Mécanique de Grenoble

B.P. 53 X, 38041 Grenoble, France

** Griffith University, Australian Environmental Studies, Brisbane, Australie

Prédiction de la courbe de succion à partir de la distribution de dimension des particules

On présente un modèle pour prédire la relation de pression effective - teneur en eau $(h(\theta))$ d'un sol à partir de la distribution de dimensions des particules. Les effets d'hystérésis et d'air occlus dans le système poreux sont pris en compte. Le fonctionnement du modèle est fondé sur l'hypothèse d'une relation directe entre le rayon de pores et le diamètre des particules correspondant, en introduisant un coefficient d'assemblage, constant pour chaque sol. La plupart des paramètres nécessaires pour le modèle sont obtenus directement par l'analyse granulométrique classique.

Les résultats relatifs à deux expériences de laboratoire montrent clairement l'existence d'une similitude de forme entre la relation $h(\theta)$ et la loi de distribution de dimension des particules.

La présente étude sera bientôt publiée sous forme d'un article dans « Soil Science».

\section{Predicting a suction curve from particle size distribution}

A model is submitted to predict the ratio of effective pressure to water content $(h(\theta))$ in soils from the distribution of particle size. Hysterisis effects and occluded air in the porous system are taken into account. Operation of the model is based on the assumed direct relation between pore radius and corresponding particle section by introducing an assembly factor that is constant for each soil. Most of the parameters required in the model are obtained directly by the usual grain size analysis.

Data obtained from two laboratory tests clearly point up the existence of similarity of shape between ratio $h(\theta)$ and the law of grain size distribution. The study is due to be published shortly as an article in "Soil Science".

\section{O.COGELS}

Département de Génie rural Université Catholique de Louvain 1348 Louvain-la-Neuve, Belgique

Effet de la variabilité à petite échelle sur la description de l'uniformité de l'irrigation

Le problème de la description de l'uniformité d'une irrigation tenant compte de la variabilité des mesures à petite échelle, c'est-à-dire à l'échelle d'influence des zones racinaires des plantes, est discuté à l'aide de mesures de distributions spatiales de lames d'eau arrosées et de teneurs en eau du sol après une irrigation effectuée par vent fort. Les schémas d'échantillonnage utilisés pour cette expérience permettent de comparer la variabilité à petite 
échelle à la variabilié totale à l'échelle de la parcelle. La dépendance spatiale des mesures est analysée à l'aide de fonctions d'autocorrélation.

\section{Effects of small scale variability on a description of uniform irrigation}

The problem of the description of irrigation uniformity, taking into account the small scale variability of the measurements, i.e. the scale of influence of the plant rootzones, is discussed with the aid of measurements of spatial distributions of water application depths and of soil water content after an irrigation with heavy wind. The sampling schemes used for this experiment allow for a comparison between small scale variability and total variability. The spatial dependance between the measurements is analysed with the aid of autocorrelation functions.

\section{G. MALLESSARD*, B. BLAVOUX* et R. GUENNELON**}

* Laboratoire de Géologie, Faculté des Sciences 84000 Avignon, France

** I.N.R.A., Station de Science du Sol,

Domaine de St-Paul

84140 Montfavet, France

Hétérogénéité en réponse à l'infiltration d'une cuve de loess sans fond, lors d'une expérience de traçage

Une expérience d'infiltration a été réalisée sur une cuve de loess de $18 \mathrm{~m}^{3}$, équipée selon deux verticales de tensiomètres et de bougies poreuses de prélèvement de la solution du sol, ainsi que d'un tube central pour sonde à neutrons.

L'eau utilisée contenait du nitrate de calcium, du chélate de fer et du tritium.

Après avoir relevé les caractéristiques initiales du matériau (succion, teneur en eau, traceurs), il a été procédé au suivi des paramètres hydrodynamiques du système et du déplacement des traceurs en profondeur, ceci pendant 350 heures après la mise en eau de la cuve.

De plus, l'évolution de la teneur en ${ }^{18} \mathrm{O}$ de l'eau du sol a été observée.

On constate que les deux verticales précitées ont des comportements tout-à-fait différents.

La singularité de la réponse de chaque verticale est analysée pour essayer de comprendre le fonctionnement de la cuve, compte-tenu d'une étude antérieure, et la représentativité de l'expérience à son échelle est discutée.

Heterogeneous response to seepage of a loess-filled A seepage experiment has been performed in a $18 \mathrm{~m}^{3}$ loess-filled tun, equipped with tensiometers and sampling porous ceramic cups (both with horizontal rods) disposed on two verticals, and a central access tube for a neutron moisture gauge.

The water used was traced with calcium nitrate, iron chelate and tritium.

The initial characteristics of the sediment (succion, moisture content, tracers) have been plotted. Then, the hydrodynamical parameters of the loess, the transfer of the tracers down to the depth and the isotopic composition $\left({ }^{18} \mathrm{O}\right)$ of the extracted waters have been observed during 350 hours after the tun was put under water. We note that each of both above-mentionned verticals has is own behaviour.

Their singular responses are analyzed to attempt to understand the working of the tun, taking into account a previous study, and the representativity of the experiment at its scale is discussed.

\section{A. GOLOVANOV et A. ZEILIGUER}

Chaire de Pédologie de l'Institut d'Amélioration des Sols, Moscou, URSS

Recherche appliquée des paramètres représentatifs des modèles mono-dimensionnels, compte tenu de la variabilité spatiale des sols

La simulation de certains processus physico-chimiques dans les sols, à l'aide de modèles déjà validés, répond à plusieurs finalités :

- choix des meilleures solutions d'amélioration du sol, pour le rendement des récoltes, pour le maintien de sa fertilité.

- étude quantitative de la solution retenue.

- prévision et gestion du déroulement de divers processus, dans le temps et dans l'espace, sous l'influence des interventions entreprises.

Cette utilisation des modèles se heurte à certaines difficultés parmi lesquelles on peut citer les conséquences de la variabilité spatiale des propriétés des sols.

Applied research on representative parameters for monodimensional models compatible with spatial variability of soils

Simulating certain physico-chemical processes in soils using previously validated models meets a number of needs e.g.:

- identifying the optimum solutions for soil improve-

ment, crop yield and enhanced soil fertility:

- quantitative analysis of solution decided on:

- projection and control of the sequencing of various processes in time and space, under the effects of the actions considered.

There are however difficulties in the way of using such models, amongst which are the consequinces of spatial variability of the soil properties.

\section{SARDIN}

Laboratoire des Sciences du Génie chimique

CNRS, 1 rue Grandville, 54042 Nancy Cedex, France

Echanges et dispersion en récupération améliorée du pétrole par voie chimique

Le taux de récupération du pétrole brut des gisements exploités atteint aujourd'hui $30 \%$ en moyenne, ce qui signifie que pour une tonne extraite, deux sont laissées en 
place. Les nouveaux procédés de récupération améliorée pourraient porter ce taux à $45 \%$.

La récupération améliorée par injection de microémulsion et de polymères est une des méthodes applicables à des gisements d'huile de densité moyenne, mais l'insuffisance de l'efficacité des produits chimiques et l'insuffisance des connaissances des mécanismes internes de la récupération au cours du transit dans la roche réservoir en limite beaucoup le domaine technique d'emploi. Les efforts de recherche portent à l'heure actuelle sur la formulation de nouvelles microémulsions et la mise au point de stratégies optimales d'injection. A cette fin, en relation avec des expériences de percolation en colonne de laboratoire et sur champ, sont en cours de mise au point des modèles de gisement tenant compte de la dynamique de l'écoulement et des interactions physicochimiques entre phases.

\section{Exchange and dispersion in enhanced oil recovery by chemical means}

The oil recovery from a producing oil field can be estimated nowadays at about $30 \%$ on an average basis, which means that when a barrel of oil has been extracted, there are still two left in the field. The recently developed enhanced oil recovery techniques could increase the rate of recovery up to $45 \%$.

The micellar-polymer process, which consists of injecting a microemulsion slug followed by a polymer buffer, can be applied to oil fields having medium gravity crude. Because of the insufficient efficiency of the chemicals used and of the lack of understanding of actual mechanisms occuring inside the reservoir rock during the flood, its field of application is restricted. The emphasis is now put on finding new microemulsion formulations and on determining which would be the best strategy for injecting the fluids. Thus numerical models, capable of simulating the transport of the species and the physico-chemical interactions between phases, are being developed for a better interpretation of laboratory experiments and field tests.

\section{MARTAUD et T.D. DINH}

\section{Institut de Mécanique des Fluides de l'Université Louis-Pasteur \\ ERA CNRS 0594, 2, rue Boussingault, 67083 Strasbourg Cedex, France}

Recherche en laboratoire sur la migration du mercure en milieu poreux saturé en relation avec un cas réel de pollution des eaux

La surveillance de la qualité de l'eau d'une rivière polluée d'une part et de l'eau de la nappe proche d'autre part, ainsi que des expériences de contrôle au laboratoire de la teneur en mercure d'une eau percolant à travers des boues mercurielles donnent une appréciation globale du phénomène de migration du mercure en milieu poreux saturé. Dans le cadre d'une recherche fondamentale où l'on exige une explication fine des mécanismes en jeu, ces travaux ont une portée limitée. Une approche visant à isoler chaque contribution à la rétention du mercure sous forme de chlorure mercurique sur une matrice poreuse a été entreprise. Elle a révélé des comportements peu habituels lors d'expériences de dispersion hydrodynamique avec échange sur des colonnes de sable. D'une part la capacité de rétention du sable pour le mercure, mesurée en essais statiques, est dépassée au cours d'essais sur colonnes, d'autre part, la forme des courbes de réponse à une injection en continu de soluté suggère l'intervention d'un phénomène de rétention particulier éventuellement propre au cas traité dans nos expériences. Comme les données manquent encore pour avancer une explication, il est prévu d'affiner les méthodes de recherche.

\section{Laboratory research on mercury migration through a saturated porous medium in relation with a field case of water pollution}

Monitoring the quality of polluted river water and groundwater and carrying out some laboratory experiments where the mercury content of water seaping through mercury bearing sludges is controled enables to appreciate the extent of the mercury migration phenomenon through a saturated porous medium. In the frame of a research plan where an accurate explanation of the mechanisms involved is needed, these experimentations are of limited interest. Further work has been undertaken, it aimed at understanding each contribution to the retention of mercury under the mercury (II) chloride form on a porous matrix. Transfer experiment in packed beds reactions of natural sand yielded unusual results. On one hand, the capacity of sand for mercury predicted form isotherms tests was surpassed in the columns tests; on the other hand, the observed shape of the breakthrough curves may lead to suspect the occurrence of a peculiar retention phenomenon, eventually depending on our frame of work. As data are still not numerous enough to give an explanation of the phenomena involved in the mercury migration, it is planned to improve our experimental scheme.

\section{B. AMBROISE et G. NAJJAR}

Laboratoire de Géographie physique ERA 569 CNRS, Institut de Géographie 43, rue Goethe - 67083 Strasbourg Cedex, France

Cartographie de l'évapotranspiration journalière en région montagneuse. Application au petit bassin du Ringelbach,

Des cartes d'évapotranspiration potentielle journalière ETP peuvent être obtenues en montagne à partir de la formule de Brochet et Gerbier (1972)

$$
E T P=m R g+n E p
$$

en cartographiant séparément l'irradiation globale $R g$ (en fonction des pentes, orientations et masques orographiques) et l'évaporation Piche $E p$ (en implantant un réseau d'évaporimètres Piche). Des cartes d'évapotranspiration réelle journalière $E T$ peuvent en être déduites en utilisant la formule de Brunet (1981)

$$
E T=E P o-(E T P-E P o) \Gamma / \Delta
$$

où $E P o$ est l'évaporation potentielle climatique. Cette méthode a été testée sur un petit bassin montagneux et a 
permis de mettre en évidence pour chacune de ces variables d'importantes variations spatiales, fonction de la structure topographique et de l'état hydrique du bassin ainsi que du type de temps. Elle donne des résultats tout à fait comparables à ceux d'autres méthodes d'estimation locale ou globale de l'ET.

\section{Mapping of daily evapotranspiration in a mountainous area - Application to the Ringelbach catchment, High Vosges (France)}

Maps of daily potential evapotranspiration ETP in a mountainous area can be obtained by using Brochet and Gerbier's formula (1972)

$$
E T P=m R g+n E p
$$

and by mapping separately the global radiation $\mathrm{Rg}$ (by taking into account the slopes, aspects and orographic masks) and the Piche evaporation Ep (by implanting a network of Piche evaporometers). Maps of daily actual evapotranspiration can be estimated by using Brunet's formula (1981)

$$
E T=E P o-(E T P-E P o) \Gamma / \Delta
$$

where EPo is the climatic potential evaporation. This mapping method has been applied to a small mountainous catchment and has revealed for each of these variables significant spatial variations related to the topography, soil moisture and weather type. Results are in good agreement with those of other local or global estimation methods of ET.

\section{S. RAMBAL}

C.E.P.E.-C.N.R.S., B.P. 5051 , 34033 Montpellier Cedex, France

Variabilité des propriétés hydrodynamiques du sol à l'échelle d'un versant karstique

Nous avons étudié les propriétés hydrodynamiques du sol d'un versant karstique, en tenant compte de leurs structures spatiales. Après un jour de drainage, la distribution de la teneur en eau semble suivre une loi bêta incomplète, celle du flux de drainage, une loi log-normale. L'analyse de la distribution conditionnelle de la teneur en eau permet d'affecter des probabilités aux caractéristiques de drainage.

Variability of the soil hydrodynamic properties at a karstic hillside scale

We have studied the soil hydrodynamic properties of a karstic hillside taking into account their spatial structures. After one day of draining, the soil water content distribution seems to follow a incomplete beta function and the drainage flux is lognormaly distributed. The analysis of the water content conditionnal distribution allows to assign probabilities to draining characteristics. 
